\title{
Enabling Interaction with Virtual Fluids and Mixed Media using a High Dexterity Hand Exoskeleton
}

\author{
Annika Schmidt ${ }^{1,2}$, Aaron Pereira ${ }^{2}$, Thomas Baker ${ }^{3}$, Benedikt Pleintinger ${ }^{2}$, Thomas Hulin ${ }^{2}$, \\ Zhaopeng Chen ${ }^{2,4}$, David A. Abbink ${ }^{5}$, Neal Y. Lii ${ }^{2}$
}

\begin{abstract}
Advances in exoskeleton technology now enable interacting with rigid objects in a virtual or remote environment using one's hand and fingertips. However, interaction with non-solid materials - such as liquids, sediments and regolith - alongside solids, can greatly extend the versatility of this technology. Rendering rigid objects adequately requires a control loop with high update rates, whereas fluid dynamics equations are computationally expensive. To accommodate this, the fluid dynamics can be simplified - particularly for fluids with high viscosity - resulting in a fast-to-calculate model to enabling haptic rendering of viscous fluids and rigid bodies simultaneously using DLR's Exodex Adam hand exoskeleton. Viscosity as a proprioceptive cue of fluids can be presented to the human through force feedback at multiple points on the human hand - fingers and palm - letting the user interact with a virtual environment in a more natural way and making the experience more immersive. We carry out two user studies to investigate the human perception abilities of virtual fluids rendered with simplified dynamics, and the discernability of different viscosity in virtual fluids compared real fluids. Results show that virtual media can give the user the perception of interacting with a fluid, even with simplified models, at a high update frequency. Furthermore, the material discernibility corresponds well to actual interaction with real viscous fluids. This shows great promise forward for haptic in-hand interaction in fluid and mixed media environments.
\end{abstract}

\section{INTRODUCTION}

Haptic cues play a key role in achieving full immersion in a virtual or remote environment, and can improve performance in virtual/augmented reality or teleoperation scenarios [1]. Haptic interfaces that apply forces or stimulate tactile senses can convey these haptic cues to the user [2]. Hand exoskeletons with many degrees of freedom (DOFs) can be used for this purpose: through natural exploration procedures involving the whole hand, interactions with a virtual or remote environment become more intuitive.

\section{A. Hand Exoskeletons}

Since the CyberGrasp System, the first commercial hand exoskeleton in the 1990s, which reflected forces to the user by changing the length of tendons [3], many new systems have been developed applying different force rendering approaches. Some use pneumatic actuation (Rutgers Master II

\footnotetext{
${ }^{1}$ Department of Informatics, Sensor Based Robotic Systems and Intelligent Assistance Systems, Technical University Munich, Garching Germany

2 Robotics and Mechatronics Center at the German Aerospace Center, Oberpfaffenhofen, Germany

${ }^{3}$ Medtronic, Dublin, Ireland

${ }_{5}^{4}$ Agile Robotics, Gilching, Germany

5 Faculty of Mechanical, Maritime and Materials Engineering at Delft University of Technology, Delft, Netherlands
}

[4], Festo Exohand [5]), servo-shifting units (Dexmo [6]) or underactuated kinematics (HEXOTRAC [7]). Next to such ungrounded devices that are worn by the user, grounded devices attached to a fixed base, such as the five-fingered Haptic Interface Robot (HIRO) [8], relieve the user from the system's weight. HIRO is mounted on a robotic arm and mirrors the human hand in appearance and motions. All these exoskeletons render forces to individual fingers, enabling more intuitive interactions in virtual or remote environments.

\section{B. Computing Fluid Forces}

While many methods have been explored to render rigid and deformable objects, the haptic rendering of fluids has been mostly neglected thus far. However, it is crucial to many application areas [9], such as training divers, underwater search and rescue, maintenance of nuclear reactors, and in general operation of robots/machines in environments where fluid forces (from e.g. mud, oil or water) are significant. A first method to compute fluid forces for haptic interactions was introduced by Baxter and Lin in 2004 [10]. They proposed a numerical method based on the classical Navier-Stokes equation of fluid motion. It calculated forces accurately, but was resource intensive. To reduce calculation effort, other approaches used pre-calculated or pre-recorded data. Dobashi et al. [11] decomposed the fluid dynamics into a nonlinear pre-computed part, and a linear part calculated online, allowing the algorithm to run at $500 \mathrm{~Hz}$. The approach was extended by Hover et al. [12] by recording arbitrary manipulations of an object in multiple viscous fluids, and interpolating the data to render the fluid interaction to a user. Although the methods reduced calculation efforts, they relied on pre-computations or recordings, which limited general applicability. To enable 6-DOF interactions with fluids of variable viscosity and for arbitrary shaped bodies, Ciro et al. [13] uses a Smoothed-Particle Hydrodynamics (SPH) simulation to represent fluid and solid bodies by particles. This approach is versatile, but again resource intensive, only realized at $60-120 \mathrm{~Hz}$. For more realistic fluid interactions, Wang and Wang proposed a hybrid method that also used SPH for the fluid, but added a flexible proxy modeled through FEM running in real time with hardware acceleration [14]. In comparison, Vines, Mora and Lee used a more simplified approach to calculate fluid forces [15]. Viscosity forces were approximated as proportional to velocity of the haptic interface point, and inertia forces were regarded as proportional to the extension of a virtual mass-spring system attached to the haptic interface point [16]. 


\section{Mixed-media Whole-hand Interaction}

All aforementioned methods of haptic fluid rendering are limited to the application of one-point probes, and it is not possible to render interactions with mixed media (fluid and solid), though many practical applications require this. To help realize a haptic interface that enables fully immersive and natural interactions with a virtual or remote environment, this paper investigates the feasibility and necessity to render fluids on a hand-arm exoskeleton, while keeping update frequency high enough (500 to $1000 \mathrm{~Hz}$ [2]) for simultaneous rendering of virtual solids. In this way, we aim to identify the role that multiple haptic interface points play in fluid interaction, and determine the required complexity of the fluid force calculations to develop future technology for a fully immersive haptic experience.

Using the recently-developed hand-arm exoskeleton Exodex Adam [17], [18] (Section III) of the the German Aerospace Center (DLR), we implement a simplified method (Section III) to render fluid forces with high update rates, similar to the approach in [15]. To test human perception capabilities of the rendered fluids we conducted two humanin-the-loop experiments. First, the perception of a rendered fluid to represent water is investigated (Section IV). Second, the discrimination abilities of the human for a variety of rendered viscosity is quantified (Section V), as humans frequently interact with fluids other than water [19].

The goal of this research is not to compare rendered virtual fluids to the perception of real fluids, as neither are the intended force calculations accurate enough, nor can residual dynamics of current mechanical systems be completely compensated for: despite feed-forward control [20] and friction compensation [21], the feeling of full immersion is still restricted by mechanical limitations. Instead, this work focuses on quantifying the ability of human users to perceive virtual media to be fluid, and their discernibility of different fluid viscosity despite simplified dynamics. The findings of the work are discussed in Section VII

\section{HAPTIC INTERFACE}

To interact with virtual fluids, we employed the novel robotic hand-arm exoskeleton Exodex Adam: a haptic interface consisting of modular robotic fingers attached at multiple points on the hand and mounted with a base on a KUKA Light Weight Robot (LWR) 4+. Three attachment points are at the operator's thumb, index, and middle fingers; two are at the palm (Fig. 11). It can sense and apply 3-DOF forces at the attachments, allowing whole-hand interaction.

Thus, in contrast to previous work, we can reflect fluid forces acting over different areas on the hand to the user through multiple interface points. In our setup, we calculate fluid forces for the whole hand and divide across attachment points in proportion to area. Forces on finger attachments are assumed to act across the entire finger area, and forces on palm points act on the palm in equal measure. The ring and little finger (in this setup not connected to the interface) are not considered. Therefore, $10 \%$ of the fluid forces are applied on each human digit, i.e. thumb, index and middle finger, and $35 \%$ acted on each palm attachment.

In our control, the LWR compensates the weight of the exoskeleton, allowing the operator to investigate the virtual fluids with a natural sweeping motion of the hand without feeling the weight of the device. The LWR control cycle time is $1 \mathrm{kHz}$; detailed control is described in [22].

The exoskeleton control is based on [23], [24], whereas the communication hardware of the exoskeleton limits its control cycle to $833 \mathrm{~Hz}$. The high-level control loop for each modular robotic finger is shown in Fig. 2. The human user $Z_{u}$ and the virtual environment $Z_{e}$ act as impedance; the haptic interface is modeled as an admittance. From the joint positions $X$ of the robotic fingers the Cartesian positions of the attachment points is found and used to estimate the human hand pose [17]. This in turn is used to calculate the virtual (fluid) forces $F_{e}$. Measuring the force the user applies on the device $F_{u}$ (different from the physical force $F$ the human uses to move their hand and arm) allows adding a feed-forward term $K$ to reduce the perceived inertia. Summing this with the rendered virtual forces, the total force to apply at the attachment with the human, $F_{c m d}$, is determined. Friction compensation as in [21] is also applied. The feed-forward gain, which helps overcoming mechanical viscosity and the large inertia due to high gear ratio would require scaling of the environment forces with the reciprocal of the gain [20]. However, since exoskeleton forces are reflected by the LWR, which has no feed-forward gain, such scaling may be perceived as an additional gain from the LWR. Hence, forces are not scaled. Despite the friction compensation, residual system dynamics due to friction and inertia were perceivable by the user, resulting in a perceived mechanical viscosity. Since this mechanical viscosity can not yet fully compensated for, it remains as an offset for the users perception when moving in free space. Any rendered viscosity of fluids is only perceivable as an addition or change to these inherent system dynamics.

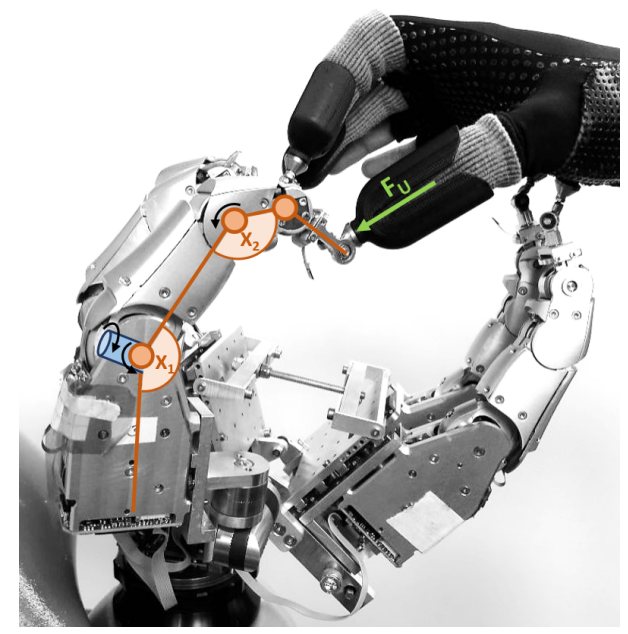

Fig. 1. Haptic interface Exodex Adam attached to operator at two palm points and three finger points (thumb, index and middle fingers). Actuated joints of the modular robotic finger are $X_{1}$ and $X_{2}$ (base and distal flexionextension) and base abduction-adduction (not shown). $F_{U}$ is the force exerted by the human on the attachment point. 


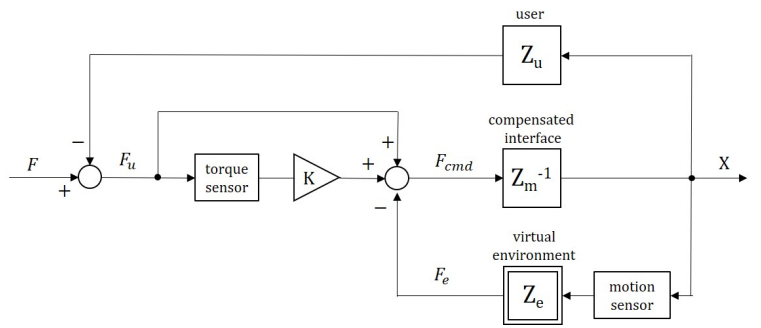

Fig. 2. The block scheme of impedance interaction with feed-forward compensation implemented for each individual robotic finger on the novel haptic interface, where $F, F_{c m d}, F_{e}$ and $F_{u}$ describe the applied forces, $K$ is a feedforward gain, $Z_{u}, Z_{m}$ and $Z_{e}$ are impedances and $X$ the joint positions.

\section{HAPTIC FLUID RENDERING}

To achieve the high update frequencies required for the interaction with different media in a virtual or remote environment, simplifications to the fluid dynamics are assumed. For accurate calculations of fluid forces acting on an emerged body complex partial differential (Navier-Stokes) equations need to be solved, which is computationally intensive [26]. Thus, a trade-off between accuracy and sufficiency needs to be made. Vines, Mora and Lee [15] identified that a key aspect to approximate perceivable fluid flow behavior is the opposing forces of the fluid to motion (drag). Inspired by this work, we propose to restrict the rendering of fluid forces for complex haptic interfaces like the used exoskeleton to only represent those drag forces (Fig. 3). For horizontal sweeping motions through a fluid, this force limitation is valid, since only negligible lift forces act on the hand when the maximum frontal area of the hand points in the direction of motion [27]. Thus, for this first investigation into the necessary complexity of the haptic interface in virtual fluids, we ask the participants of the user studies to only execute this sweeping motion. This can be expanded in future work. Further, buoyancy can be neglected, as water and most viscous fluids are of similar density as the human body [28]. The contribution of drag forces, which act against the direction of motion [29] can be calculated using

$$
F_{D}=\frac{1}{2} \rho v^{2} A C_{D},
$$

where $v$ refers to the moving velocity of the flow or object, i.e. the human hand in a motionless fluid, here determined by sensors of the haptic interface. The reference area $A$ refers to the maximum projected area of the human hand, here set to $0.0148 \mathrm{~m}^{2}$ as proposed by Sato [30]. The fluid density $\rho$

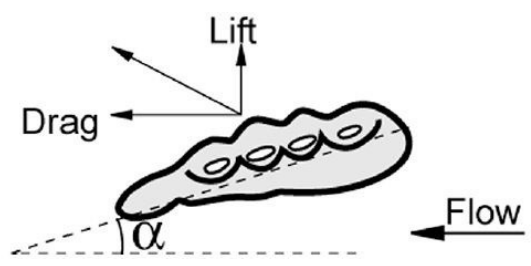

Fig. 3. Human hand subjected to flow with acting lift and drag forces, which are dependent on the angle of attack $\alpha$ [25]. is assumed to be $1000 \mathrm{~kg} / \mathrm{m}^{3}$, which equals the density of water and many viscous silicones [31]. The drag coefficient $C_{D}$ is a body specific dimensionless number that varies with the type of flow and the form and orientation of the body. For water, the drag coefficient $C_{D}$ of the human hand has been determined in various studies [12], [25], [27], [30]. Even though the magnitude range varies depending on the applied procedures and measurement methods, a distinctive curve shape can be observed, which depends on the angle of attack $\alpha$ for the flat hand with extended fingers. Fitting a sinusoid to the drag coefficient curve in [30] leads to the relationship

$$
C_{D}=-0.3105 \cdot \cos (2 \alpha)+0.5794 \text {. }
$$

With the position encoders of the haptic interface the hand's $\alpha$ can be determined in every moment during the interaction. Substituting (2) into (1) leads to an analytic expression for the drag forces of water, which can be calculated in real time. This relationship is used for the first human user study.

For fluids other than water, finding the drag coefficient $C_{D}$ value is not so trivial, as it was not specifically investigated for the human hand. However, when considering fluids with higher viscosity, further simplifications can be made as the flow properties depend on the Reynolds number [29]:

$$
R e=\frac{\rho v L}{\mu},
$$

where $L$ is a characteristic length of the emerged body and $\mu$ the viscosity. Toussaint proposed the characteristic length of the human hand to be the chord length $(\approx 0.1 \mathrm{~m})$ [25]. When assuming the hand velocity to be $<1 \mathrm{~ms}^{-1}$ for exploratory motions in fluids, and the viscosity $\mu$ to be in a range of $2-100 \mathrm{~Pa} s$ for viscous fluids, a Reynold's number of 1-10 is determined. Liquids in such regimes show only insignificant effects of inertia and are dominated by internal friction, leading to a laminar flow with negligible turbulence [29]. Assuming the human hand flat without finger spread and the flow around it to be linear and without flow separation due to the low Reynolds number, the flow regime can be assumed to be Stokes Flow. This results in the following simplification for the drag coefficient [29]:

$$
C_{D}=\frac{24}{R e} .
$$

Substituting this into (1) yields a simple analytical expression for drag force, similar to the equation proposed by Vines, Mora and Lee to render the viscosity [15]. This can be computed quickly, allowing high update frequencies for the real time robot interaction. This relationship is used for the second user study. Solid objects in the current setup are rendered using the god-object method [32].

\section{STUdy 1: Perception OF VIRTUAL WATER}

Water is the most common fluid that humans interact with. Thus, a first user study is carried out to investigate if virtual water with simplified flow properties can be perceived as such using the Exodex Adam as haptic interface, taking into account the residual system dynamics. Participants include 13 right-handed people (4f, 9m) aged between 21 and 29. 


\section{A. Methods}

The experiment setup to investigate the perception of virtual fluids is shown in Fig. 4 and in the linked vided 1 . The user faces the robotic interface with the right hand attached to the exoskeleton. To accustom to the mentioned residual dynamics of the robotic system (i.e. the inertia and friction) perceivable in free space, each participant first has time to freely explore the setting without any rendered forces. No visual representation is shown to the user to focus on the haptic perception without the influence of other senses. Afterward, three example trials are given to ensure the participants understand the task. For interaction with the virtual fluids during the experiments, the participants are asked to carry out a horizontal sweeping motion while imagining a wall of virtual fluid to be in front of them. The stroke amplitude and velocity are not specified, but left to the user's discretion, to enable a more natural interaction. Verbal answers of the participants are noted by the experimenter, while system data, such as occurring and rendered torques as well as hand and finger trajectories are recorded with a sampling time of $1 \mathrm{kHz}$. Additionally, videos of the experiment are recorded for the purpose of reviewing the human-robot interaction.

To quantify the perception of virtual water, the viscosity of the rendered drag forces are compared to the viscosity perceived by the user. For this, the calculated forces defined through (1) and (2) are scaled with a gain between 0 and 4 (in steps of 0.5 ) to find the scaling factor for which the participants confirm to perceive the rendered fluid as water. The scaling factor was quantified through a combination of magnitude estimation and magnitude production, due to an expected bias inherent to both methods [33]. Using both methods can cancel the bias to obtain more accurate results. During the magnitude estimation, the user is presented with differently scaled forces, and has to estimate the intensity of each stimulus, where $100 \%$ defines the feeling of water. Three trials are carried out, within each the 9 scaling factors are presented in pseudo-randomized order. During the production part of the experiment, the user is asked to set the given stimulus until the virtual fluid is perceived to have the viscosity of water $(100 \%)$, starting from a scaling factor in the range of $0-4$. Each scaling factor is used as starting point once in randomized order, leading to a repetition of 9 times. In a second and third trial, the user repeats the procedure adjusting the stimulus to be perceived as $50 \%$ and $200 \%$ of the viscosity of water, respectively. Whether the participants started with the magnitude estimation or production was randomly assigned.

For the analysis of the data, a curve was fitted to the results of each participant for the part of magnitude estimation and production. To find the overall relationship between the given stimulus and the perceived viscosity, the mean curve for both experiment parts was calculated and fitted to a power function of the form

$$
S=k I^{a}
$$

${ }^{1}$ https://www.youtube.com/watch?v=rrQJJPXCffg

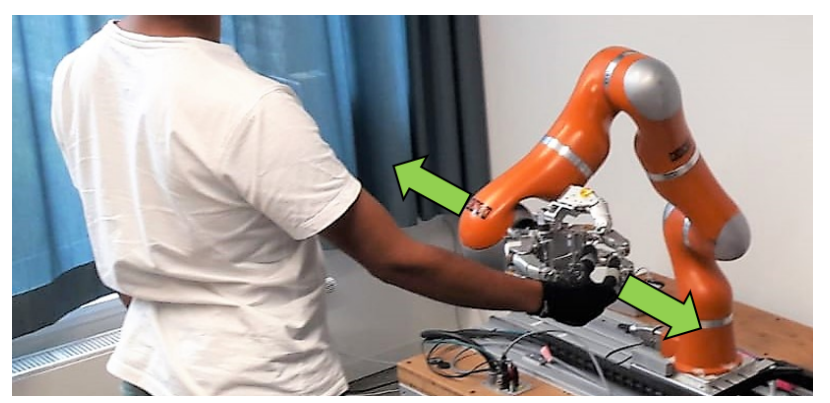

Fig. 4. Test setup for user studies with the participant positioned in front of the robotic device. The human hand is attached to the hand exoskeleton mounted on a KUKA LWR. Green arrows indicate the specified direction of motion to investigate the virtual fluid.

where $S$ is the sensation magnitude, i.e. the perceived viscosity by the user, and $I$ the set intensity of the stimulus, i.e. the actual rendered viscosity. The exponent $a$ and the constant $k$ parameterize the relationship between the two. After the experiment, a questionnaire is filled out rating the haptic interface and the perceptions during the task.

\section{B. Results}

When taking into account the residual mechanical viscosity due to the device dynamics, all participants are able to identify the rendered media to be fluid. Consistent results for the scaling to perceive water could be obtained. Carrying out a regression analysis for the magnitude estimation (Fig. 57) showed a moderate to strong correlation for all participants, except the first. The curve of this person appeared offset from the main trend, but is not disregarded as the video review does not show abnormalities. The difference might just relate to variation in individual perception. For the magnitude production (Fig. 5b), similar curves can be found for all participants, but a varying steepness can be seen. However, for all participants, the ratios of the set scaling factors appeared to be constant, i.e. when the participants set a higher stimulus intensity for the perception of $50 \%$ water viscosity, they also set the stimuli proportionally higher for $100 \%$ and $200 \%$ perceived viscosity of water. A relatively constant value wass found throughout the participants for the ratio between 100 and $50 \%$ virtual water viscosity $(1.97 \pm 0.34(\mathrm{sd}))$ and 200 and $100 \%(1.78 \pm 0.55(\mathrm{sd}))$. Fitting a power function curve between the averaged points per participant for magnitude estimation and production shows the expected regression bias between the two methods [33] (Fig. 56). To compensate the inherent bias, another power function is fitted between the two curves. This leads to a general relationship between the given and the perceived stimulus of:

$$
S=0.5145 \cdot I^{0.9021} \text {. }
$$

This indicates a near-linear relationship between the given and perceived intensity of the viscosity stimulus. It also shows that the forces were perceived half as intense as calculated, meaning a gain of 2 had to be applied to the rendered drag forces so that the user would identify the rendered fluid to be virtual water. 

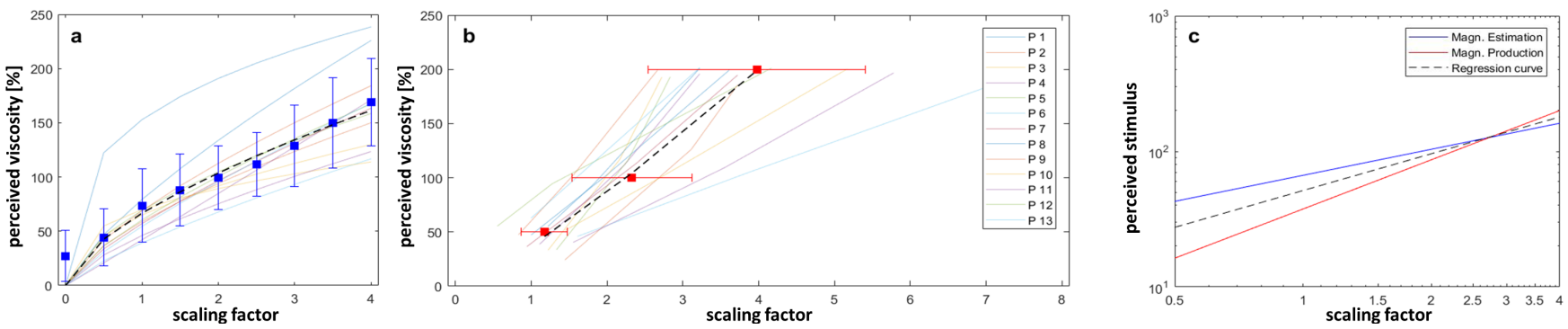

Fig. 5. Results of Study 1, showing the relationship between the perceived viscosity of virtual water to the set scaling factor in (a) magnitude estimation and (b) production. Each colored line corresponds to one participant. A power function is fitted for each procedure to obtain the general relationship. The averaged values are depicted by squares $(\mathrm{n}=13)$ with error bars indicating the standard deviation. $(\mathrm{c})$ shows the comparison of the fitted power functions of the two procedures with a fitted regression curve.

Observing the investigation procedure applied by the participants to inspect the virtual fluid, similar sweeping motions are found for all participants. Although neither the stroke amplitude nor moving velocity were specified, the stroke width $(0.260 \mathrm{~m} \pm 0.056 \mathrm{~m}$ (sd) $)$ as well as the maximum hand velocity $(0.4409 \mathrm{~m} / \mathrm{s} \pm 0.0830 \mathrm{~m} / \mathrm{s}(\mathrm{sd}))$ are constant throughout all participants. Analyzing the recorded joint angles of the exoskeleton fingers and the video recordings, it appears that the hand movement is led by the palm, pushing the attached exoskeleton fingers when moving forward, and pulling during the backward motion (see video link). The thumb, index and middle fingers of the human seem to not be used extensively. They only counteract the motion of the palm, spreading outward when pushing forward, and being flexed inward when moving backward. They move little otherwise. Despite the high update frequencies, no instability is observed during the fluid rendering, i.e. no unwanted vibrations or perturbations could be felt.

\section{STUdy 2: ViRTUAL Viscosity Discrimination}

In order to investigate the human perception abilities of virtual fluids with varying viscosity, a second user study is carried out that replicates, in parts, a study of Bergmann Tiest et al. [34]. That study showed that humans are able to clearly discriminate viscosity differences above $2 \mathrm{~Pa} s$ when stirring a real fluid with a rod. The investigated viscosity groups for which significant differences are found (group D and $\mathrm{E}$ in [34]) are implemented as virtual fluids, and the human's ability to differentiate them is inspected.

Again, 13 right-handed participants $(9 \mathrm{~m}, 4 \mathrm{f})$ are included in the study, but different from those in the first experiment.

\section{A. Methods}

To quantify the perception of different viscosity, the setup and preparations are identical to those explained for the first user study (Fig. 4). Inspired by the research in [34], the method of constant stimuli is applied to identify the human discrimination abilities of virtual viscosity. The virtual fluids are rendered using equation (1) and (4), where $\mu$ defines the viscosity of the rendered fluid, and is set to the values stated by Bergmann Tiest et al. [34] for viscosity group D and E. An additional step is added for each group below and above the reference viscosity, to get a better estimation of the psychophysical curve progression (Tab. 1). A two-alternative forced-choice procedure is used, where the rendered reference viscosity $(R)$ is presented first, followed by a test viscosity, which must be judged to be of higher viscosity or not. Each test stimuli $\left(T_{1}-T_{8}\right)$ is presented once per trial in a random order. Five trials are carried out for both viscosity groups. The order in which the groups are presented is assigned to the users at random. To quantify the discrimination abilities, the percentage of times that each test stimulus is perceived as thicker than the reference is recorded. Ideally, for lower than the reference, this percentage should tend toward $0 \%$, and for higher ones towards $100 \%$. Between the extreme points a psychometric curve can be fitted, where the steepness of the curve indicates the discrimination threshold:

$$
f(x)=50 \%+50 \% \cdot \operatorname{erf}\left(\frac{\log (x / p)}{\sqrt{2} \log (w+1)}\right),
$$

where $\operatorname{erf}$ is the error function, $p$ the reference viscosity and $w$ the Weber fraction as a free parameter.

At the end of the experiment, the participants are asked to fill out the same questionnaire as used in the first user study.

\section{B. Results}

All participants are able to identify differences in viscosity for the rendered virtual fluids. Weber fractions similar to the ones obtained by Bergmann Tiest et al. [34] are determined. Plotting the perceived thickness for each rendered viscosity in groups D and E shows for all participants typical psychometric curves (Fig. 6). However, it is found that the determined Weber fractions per participant for viscosity group $\mathrm{D}$ is significantly higher $(\mathrm{n}=13$, t-test, $p<0.001)$

TABLE I

APPLIED VISCOSITIES IN $\mathrm{Pa} s$ AS PROPOSED IN [34] FOR GROUP D AND E. $T_{i}$ ARE THE TEST STIMULI ( $T_{4}$ AND $T_{5}$ ADDED to [34]); $R$ IS THE REFERENCE FOR EACH RANGE.

\begin{tabular}{ccc|ccc}
\hline Range & D & E & Range & D & E \\
\hline$T_{1}$ & 1.093 & 10.100 & $T_{5}$ & 2.203 & 17.505 \\
$T_{2}$ & 1.237 & 11.158 & $T_{6}$ & 2.553 & 18.950 \\
$T_{3}$ & 1.646 & 13.830 & $T_{7}$ & 3.185 & 23.200 \\
$T_{4}$ & 1.7495 & 14.945 & $T_{8}$ & 3.883 & 29.335 \\
\hline$R$ & 1.853 & 16.060 & & & \\
\hline
\end{tabular}




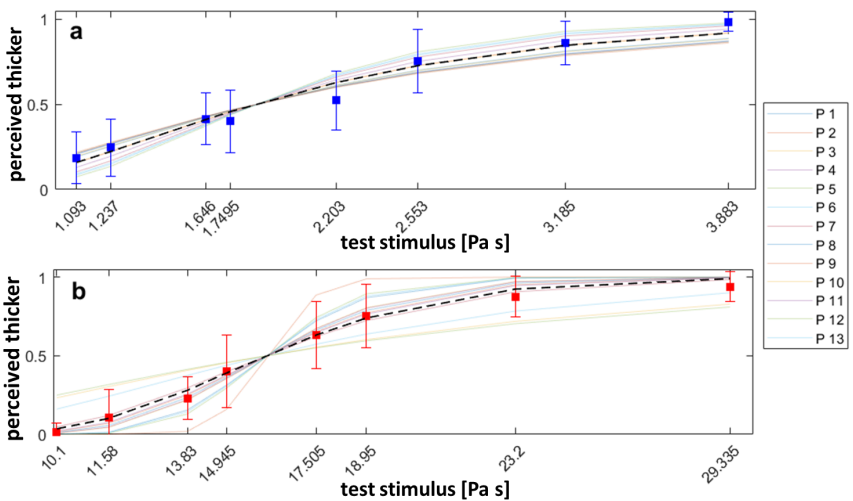

Fig. 6. Results of Study 2 giving a fitted psychometric curve defined through the Weber fraction for (a) viscosity group D and (b) E over all participants $(n=13)$ and for the mean data with the error bars indicating the standard deviation.

than for group E. Averaging the Weber fraction over all participants lead to values of $w_{D}=0.70$ for group D and $w_{E}=0.29$ for group E. This matches the findings in [34] for group E, but shows that for group D the participants are not able to discriminate the virtual viscosity well, which differed from the findings of Bergmann Tiest et al [34].

Again, overall similar investigation procedures are observed for all participants with little variation in stroke width $(0.553 \mathrm{~m} \pm 0.115 \mathrm{~m}(\mathrm{sd}))$ and maximum hand velocity $(0.413 \mathrm{~m} / \mathrm{s} \pm 0.061 \mathrm{~m} / \mathrm{s}(\mathrm{sd}))$. The overall applied velocity complies with the results from the first user study, but the stroke with iss significantly higher. Comparing the velocities within this second user study for group D and E, a significant drop in maximum applied velocity could be seen with values of $0.451 \mathrm{~m} / \mathrm{s} \pm 0.063 \mathrm{~m} / \mathrm{s}$ (sd) and $0.375 \mathrm{~m} / \mathrm{s} \pm 0.064 \mathrm{~m} / \mathrm{s}$ for group $\mathrm{D}$ and $\mathrm{E}$, respectively $(\mathrm{n}=11$, t-test, $p=0.01)$. Regarding the designated hand motions during the experiment, again the same motion pattern as for the first user study could be found with the palm dominating the interaction with the exoskeleton.

\section{PReliminary MiXed Media INTERACTION}

To test rendering different media within the same algorithm and update rate, at the end of both user studies, participants are presented with a virtual solid and fluid medium. Participants are asked to hold their hand at head-height with the palm facing downwards, then slowly move their hand down until they feel resistance and describe the feeling they perceive (see video link). In one case a fluid is rendered, which the user should perceive in a change of viscosity from free space to fluid. In the second case, within the same rendering algorithm, a solid horizontal wall is rendered with the god-object method. The order in which the two cases are presented is randomly chosen. This preliminary tests show that it is possible to render solids and fluids within the same rendering algorithm and interactively switch between the two. All 26 participants could clearly distinguish between the two media. All but one described the rendered virtual wall to be "solid" or "hard", while describing the virtual fluid to be distinctively different using words such as "gooey". The questionnaires of the two studies show that the participants judged the realism of the interaction with the virtual fluid to be $6.15 / 10$ (s.d 1.46). The users responded favorably on the perception afforded by the system. To further enhance system performance, 7 of the 13 participants recommended more tactile feeling, e.g. pressure, along the hand's surface, whereas 3 others suggested adding temperature cues would add to the realism.

With a smaller number of participants, as part of work in progress, we tested rendering both media (solid and fluid) simultaneously. A solid shape, e.g. a sphere, was submerged in a fluid. The users were able to perceive the shape of the object with the finger tips of the exoskeleton, while feeling the fluid viscosity through the rendering on the LWR. This is shown in Fig. 7 and in the linked video

\section{DISCUSSION}

In this research, we examined the feasibility and necessity to use a complex haptic interface, the DLR's Exodex Adam, to interact with virtual fluids and mixed media (fluids and solids). Due to simplifications of the fluid dynamics, i.e. the limitation to drag forces, the haptic rendering algorithm could run with high update rates, allowing simultaneous rendering of virtual solid objects in the same control loop and the force reflection on multiple interface points. Although the applicability in this stage remains to be extended, we show it to be feasible to stably render mixed media forces on a complex interface. This opens the opportunity to explore a richer virtual environment in an intuitive way.

The user studies suggest that the higher the viscosity, the finer the perception is experienced by the user. In the first experiment the results shows that a scaling factor of about 2 of the calculated fluid forces is necessary for the user to confirm the perception of virtual water. Meanwhile, the second study indicates that for higher viscosity (group E), the differentiation abilities of the humans match the findings in [34] for real fluids. The reason for this is most likely the inherent mechanical viscosity remaining in the haptic interface, i.e. friction and inertia of the robot joints. Only when the forces from the virtual fluid viscosity were perceivably

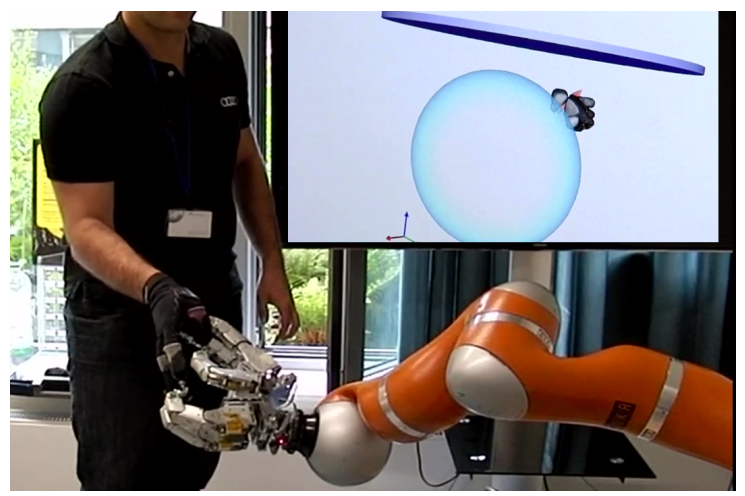

Fig. 7. Subject interacting with solid sphere submerged in a fluid. A visualisation of the virtual environment and the estimated position of the subject's hand is shown, top right. 
higher than those from the residual system dynamics, the user was able to clearly identify the media to be fluid.

This emphasizes how much of a challenge the rendering of fluids is. Not only resource intensive calculations are necessary for accurate representation of fluids, but it should be paired with a high dexterity haptic input system that can sufficiently compensate for mechanical friction and inertia. Nonetheless, the user studies show how adaptive the user can be. Although the feeling of virtual water is not identical to the physical counterpart, taking into account the system dynamics of the haptic interface, the user is able to identify and accept a virtual fluid to be water. This indicates that for most applications, a simplified fluid force approximation, such as is implemented here, may already suffice to increase the application possibilities in e.g. a teleoperation scenario and contribute to a more immersive experience.

The detailed analysis of the video recordings during both user studies show that all participants apply a similar exploration procedure and hand motion - shown in the linked video - in which the palm leads the motion, and the fingers are barely moved. Paired with the participant's feedback about the fluid interaction, this indicates that the user's perception of the fluid is dominated by the larger motions generated from the arm, rather than the fingers. This is particularly interesting: While the motion in the fluid can be perceived through the arm and palm, the fingers are free to perceive forces of manipulating solid objects in a mixed media environment. This observation also validates the assumed importance of incorporating attachment points to the human palm in the design of Exodex Adam [18]. This points to a need for more detailed analysis of natural exploration procedures in fluids, and at the in-hand level to more closely study user perceptions in fluid and mixed media environment, e.g. a specifically designed set of finger and palm motions to extract user feedback.

The findings point to the need for a full hand-arm system for immersive haptic user interaction. A complex interface may not be needed for mere perception of viscosity alone. However, an exoskeleton hand-arm system greatly extends application possibilities, as the in-hand capabilities afforded at the finger level give the user the option for simultaneous dexterous motions/tasks. Since the goal is a universal haptic interface that can be used for a variety of tasks including grasping and manipulating solid (virtual) objects, a highDOF haptic interface with multiple interface points on the hand remain necessary.

In the questionnaires, most users report that the feeling of wetness an other cutaneous cues like pressure are missing as an essential key property to perceive fluids. While those are hard to virtually replicate, in a future implementation the benefit of adding tactile cues should be investigated, e.g. by combining the vibrotactile feedback proposed by Ciro et al. [35] with the force feedback proposed in this study.

\section{CONCLUSION}

This work shows the feasibility to implement perceivable fluid forces in an exoskeleton, and paves the way to investigate the integration possibilities of virtual fluids in more complex setups involving haptic interfaces with multiple interface points. Although in the proposed work, only simplified fluid dynamics can be rendered, the cues are sufficient for humans to identify a medium to be fluid and even discriminate different fluids when the viscosity is sufficiently high. It also demonstrates the possibility to render more complex virtual or remote environments even with a haptic interface that has multiple interface points. Finally, combining the perception of virtual fluids with the interaction of virtual solid objects opens up new possibilities for more immersive interactions, which can eventually aid a wide array of virtual reality and teleoperation applications.

\section{REFERENCES}

[1] J. Artigas, R. Balachandran, C. Riecke, M. Stelzer, B. Weber, J.-H. Ryu, and A. Albu-Schaeffer, "Kontur-2: force-feedback teleoperation from the international space station," in IEEE Int. Conf. Robotics and Automation, 2016, pp. 1166-1173.

[2] B. Hannaford and A. M. Okamura, "Haptics," in Springer Handbook of Robotics, B. Siciliano and O. Khatib, Eds. Springer International Publishing, 2016, pp. 1063-1084.

[3] (2018, Jan.) Cyberglove-systems website. [Online]. Available: http: //www.cyberglovesystems.com/cybergrasp/

[4] M. Bouzit, G. Burdea, G. Popescu, and R. Boian, "The rutgers master ii-new design force-feedback glove," IEEE/ASME Trans. Mechatron., vol. 7, no. 2, pp. 256-263, 2002.

[5] (2020, Mar) Festo exohand. [Online]. Available: https://www.festo. $\mathrm{com} / \mathrm{group} / \mathrm{de} / \mathrm{cms} / 10233 . \mathrm{htm}$

[6] X. Gu, Y. Zhang, W. Sun, Y. Bian, D. Zhou, and P. O. Kristensson, "Dexmo: An inexpensive and lightweight mechanical exoskeleton for motion capture and force feedback in vr," in Proc. 2016 CHI Conf. on Human Factors in Computing Systems. ACM, 2016, pp. 1991-1995.

[7] I. Sarakoglou, A. Brygo, D. Mazzanti, N. G. Hernandez, D. G. Caldwell, and N. G. Tsagarakis, "Hexotrac: A highly under-actuated hand exoskeleton for finger tracking and force feedback," in Proc. IEEE/RSJ Int. Conf. Intell. Robots and Systems, 2016, pp. 1033-1040.

[8] T. Endo, H. Kawasaki, T. Mouri, Y. Ishigure, H. Shimomura, M. Matsumura, and K. Koketsu, "Five-fingered haptic interface robot: Hiro iii," IEEE Trans. Haptics, vol. 4, no. 1, pp. 14-27, 2011.

[9] P. Xia, "New advances for haptic rendering: state of the art," The Visual Computer, vol. 34, no. 2, pp. 271-287, 2018.

[10] W. Baxter and M. C. Lin, "Haptic interaction with fluid media," in Proc. Graphics Interface, 2004, pp. 81-88.

[11] Y. Dobashi, M. Sato, S. Hasegawa, T. Yamamoto, M. Kato, and T. Nishita, "A fluid resistance map method for real-time haptic interaction with fluids," in Proc. ACM Symp. on Virtual Reality Software and Tech., 2006, pp. 91-99.

[12] R. Hover, G. Kósa, G. Szekly, and M. Harders, "Data-driven haptic rendering - from viscous fluids to visco-elastic solids," IEEE Trans. Haptics, vol. 2, no. 1, pp. 15-27, 2009.

[13] G. Cirio, M. Marchal, S. Hillaire, and A. Lecuyer, "Six degrees-offreedom haptic interaction with fluids," IEEE Trans. Visualization and Computer Graphics, vol. 17, no. 11, pp. 1714-1727, 2011.

[14] Z. Wang and Y. Wang, "Haptic interaction with fluid based on smooth particles and finite elements," in Int. Conf. on Comput. Science and Its Applications. Springer, 2014, pp. 808-823.

[15] M. Vines, J. Mora, and W.-S. Lee, "Haptic display of 3d liquids for interactive applications," in Proc. IEEE Games Innovations Conf., 2009, pp. 140-148.

[16] — "Real-time haptic display of fluids," in Proc. 2nd Canadian Conf. on Computer Science and Software Engineering, 2009, pp. 149153.

[17] A. Pereira, G. Stillfried, T. Baker, A. Schmidt, A. Maier, B. Pleintinger, Z. Chen, T. Hulin, and N. Y. Lii, "Reconstructing human hand pose and configuration using a fixed-base exoskeleton," in IEEE Int. Conf. Robotics and Automation, 2019, pp. 3514-3520.

[18] N. Y. Lii, G. Stillfried, Z. Chen, M. Chalon, B. Pleitinger, and A. Maier, "Handexoskelett sowie roboterarm mit solchem handexoskelett," German Patent DE102017220 996.8, 23-11-2017, pending. 
[19] Y. Nonomura, T. Miura, T. Miyashita, Y. Asao, H. Shirado, Y. Makino, and T. Maeno, "How to identify water from thickener aqueous solutions by touch," J. Royal Society Interface, vol. 9, no. 71, pp. 1216-1223, 2012.

[20] J. J. Gil and E. Sanchez, "Control algorithms for haptic interaction and modifying the dynamical behavior of the interface," in $2 n d$ Int. Conf. Enactive Interfaces, 2005, pp. 17-18.

[21] L. Le Tien, A. Albu-Schaffer, A. De Luca, and G. Hirzinger, "Friction observer and compensation for control of robots with joint torque measurement," in Proc. IEEE/RSJ Int. Conf. Intell. Robots and Systems, 2008, pp. 3789-3795.

[22] K. Hertkorn, T. Hulin, P. Kremer, C. Preusche, and G. Hirzinger, "Time domain passivity control for multi-degree of freedom haptic devices with time delay," in Proc. IEEE Int. Conf. Robotics and Automation, 2010, pp. 1313-1319.

[23] Z. Chen, N. Y. Lii, T. Wimboeck, S. Fan, M. Jin, C. H. Borst, and H. Liu, "Experimental study on impedance control for the five-finger dexterous robot hand DLR-HIT II," in Proc. IEEE/RSJ Int. Conf. Intell. Robots and Systems, 2010, pp. 5867-5874.

[24] Z. Chen, N. Y. Lii, T. Wimboeck, S. Fan, and H. Liu, "Experimental evaluation of cartesian and joint impedance control with adaptive friction compensation for the dexterous robot hand dlr-hit ii," Int. J. Humanoid Robotics, vol. 8, no. 04, pp. 649-671, 2011.

[25] H. Toussaint and M. Truijens, "Biomechanical aspects of peak performance in human swimming," Animal Biology, vol. 55, no. 1, pp. $17-40,2005$.
[26] J. D. Anderson and J. Wendt, Computational fluid dynamics. Springer, 1995, vol. 206.

[27] J. Van Houwelingen, S. Schreven, J. B. Smeets, H. J. Clercx, and P. J. Beek, "Effective propulsion in swimming: grasping the hydrodynamics of hand and arm movements," J. Applied Biomech., vol. 33, no. 1, pp. 87-100, 2017.

[28] I. P. Herman, Physics of the human body. Springer, 2016.

[29] B. Lautrup, Physics of continuous matter: exotic and everyday phenomena in the macroscopic world. CRC Press, 2004.

[30] Y. Sato and T. Hino, "A computational fluid dynamics analysis of hydrodynamic force acting on a swimmer's hand in a swimming competition," J. Sports Sci. \& Medicine, vol. 12, no. 4, p. 679, 2013.

[31] (2020, Jan.) Wacker chemie ag - solid and liquid silicone rubber-material and processing guidelines. [Online]. Available: https://www.wacker.com/h/medias/6709-EN.pdf

[32] C. B. Zilles and J. K. Salisbury, "A constraint-based god-object method for haptic display," in IEEE/RSJ Int. Cong. Intell. Robots and Sys., vol. 3, 1995, pp. 146-151.

[33] S. S. Stevens, "Problems and methods of psychophysics." Psychological Bulletin, vol. 55, no. 4, p. 177, 1958.

[34] W. M. Bergmann Tiest, A. C. Vrijling, and A. M. Kappers, "Haptic discrimination and matching of viscosity," IEEE Trans. Haptics, vol. 6 , no. 1, pp. 24-34, 2013.

[35] G. Cirio, M. Marchal, A. Lécuyer, and J. R. Cooperstock, "Vibrotactile rendering of splashing fluids," IEEE Annals of the History of Computing, no. 01, pp. 117-122, 2013. 